Proom, H. \& Knight, B. C. J. G. (1955). J. gen. Microbiol. 13, 474-480

\title{
The Minimal Nutritional Requirements of Some Species in the Genus Bacillus
}

\author{
BY H. PROOM \\ Wellcome Research Laboratories (Biological Division), Beckenham, Kent \\ AND B. C. J. G. KNIGHT \\ Department of Microbiology, The University of Reading
}

\begin{abstract}
SUMMARY: The characteristic nutritional patterns were determined for seven species or varieties of the genus Bacillus. Six strains of Bacillus cereus var. anthracis grew in a complex but defined amino acid medium + thiamine. Three strains of the insect pathogen $B$. cereus var. thuringiensis had the same nutritional pattern as the parent species $B$. cereus and grew in a seven amino acid medium. Of twenty strains of $B$. firmus, ten grew on the seven amino acid medium + biotin while the growth of five other strains was markedly affected by the addition of thiamine. Two of the remaining five strains required thiamine on first isolation, but soon dispensed with it on subculture, while the other three strains continued to require thiamine. Six strains of $B$. lentus grew in the seven amino acid medium + biotin + thiamine + urea. When the seven amino acid medium was replaced by the complex amino acid medium three of these strains of $B$. lentus grew without the addition of urea, and the remaining three strains required either urea or ammonium chloride. The sixteen strains of $B$. coagulans all grew in the complex amino acid medium + biotin + thiamine. The three strains of $B$. pulvifaciens grew in the seven amino acid medium + biotin.

The twenty-seven strains of Bacillus circulans formed a nutritionally heterogeneous group. Eight strains were maintained in serial subculture in broth but could not be grown in defined media. One strain grew in the ammonia basal medium alone, others required added biotin or added biotin + thiamine and others required amino acid media + biotin + thiamine.
\end{abstract}

In our previous survey of the nutritional requirements of mesophilic species in the genus Bacillus (Knight \& Proom, 1950) a number of species was either not examined or difficulties were encountered in determining their exact nutritional requirements. This supplementary paper fills some of the gaps left in our previous work.

\section{METHODS}

Strains. On isolation, or receipt from other collections, the strains were freeze-dried and deposited in the culture collection at the Wellcome Research Laboratories; in the text the reference $\mathrm{CN}$ refers to the Catalogue Number in this collection. Where the strain was not isolated by us the reference number of the sender is given in brackets. The strains after drying were tested for purity and identity by the methods described by Smith, Gordon \& Clark (1952) and by Knight \& Proom (1950).

Nutritional requirements. The methods used were similar to those described by Knight \& Proom (1950). For a detailed description reference should be made to that publication, but for convenience the salient points are here given. 
The usual precautions were taken to exclude contamination by unwanted nutrients. The starting-point of an experiment was a freeze-dried culture from the collection. At least five serial subcultures were made in defined media to exclude the possibility of carry-over of essential nutrients. At the conclusion of each experiment the final culture was checked for purity and identity. To exclude the possibility of selection of less-exacting variants the original strain from the collection was tested directly for its ability to grow on the medium finally selected as satisfactory for the given organism.

The media were dispensed in $5 \mathrm{ml}$. lots in $6 \times 1 \mathrm{in}$. rimless glass tubes closed by loosely fitting aluminium caps. The cultures were incubated at the required temperature in a sloped position to give good aeration. The initial inoculum was taken from an agar slope with a small loop; subsequent inoculations were made with a small loop.

\section{MEDIA}

Ammonia basal salt medium. $\mathrm{KH}_{2} \mathrm{PO}_{4}, 1 \cdot 5 \mathrm{~g}$.; $\left(\mathrm{NH}_{4}\right)_{2} \mathrm{HPO}_{4}, 7 \cdot 0$ g.; $\mathrm{MgSO}_{4}$. $7 \mathrm{H}_{2} \mathrm{O}, 0 \cdot 5 \mathrm{~g} . ; \mathrm{CaCl}_{2} \cdot 2 \mathrm{H}_{2} \mathrm{O}, 0 \cdot 3 \mathrm{~g}$.; $\mathrm{MnSO}_{4} .4 \mathrm{H}_{2} \mathrm{O}, 0 \cdot 04$ g.; $\mathrm{FeSO}_{4} .7 \mathrm{H}_{2} \mathrm{O}, 0 \cdot 025 \mathrm{~g}$; ammonium molybdate, $0.002 \mathrm{~g}$. These compounds were dissolved in distilled water to 1 l. and the $\mathrm{pH}$ value adjusted to $7 \cdot 6$ with $\mathrm{NaOH}$. The solution was boiled, filtered through paper and sterilized for $20 \mathrm{~min}$. at $115^{\circ}$.

Seven amino acid medium. Ammonia basal salt medium supplemented with the following amino acids in the concentrations shown $(\mathrm{mg} . / \mathrm{ml}$.): L-asparagine, 0.4; L-proline, $0 \cdot 1$; L-leucine, 0.57 ; DL-alanine, 0.38 ; L-glutamic acid, 1.4; DL-serine, $0 \cdot 12$; DL-methionine, $0 \cdot 06$. The amino acid mixture was prepared separately by solution in distilled water at 10 times these concentrations. It was filtered through paper and sterilized by Seitz filtration. It was then added aseptically to the ammonia basal salt medium to give the required concentration of amino acids.

Complex amino acid medium. Ammonia basal salt medium supplemented with the following amino acids in the concentrations shown (mg./ml.): DLalanine, 0.38 ; DL-aspartic acid, 0.89 ; L-arginine $\mathrm{HCl}, 0.3$; L-cystine, 0.02 ; L-glutamic acid, 1.4; glycine, $0 \cdot 17$; L-histidine $\mathrm{HCl}, 0.24$; DL-isoleucine, 0.76 ; L-leucine, 0.57 ; L-lysine $\mathrm{HCl}, 0.24$; DL-methionine, 0.06 ; L-proline, 0.11 ; DLserine, $0 \cdot 12$; DL-threonine, $0 \cdot 10$; L-tyrosine, $0 \cdot 06$; DL-valine, $0 \cdot 15$. The amino acid mixture was prepared separately by solution in distilled water at 10 times the required concentration, filtered through paper and sterilized by Seitz filtration. It was then added aseptically to the ammonia basal medium to give the required concentration.

\section{RESULTS}

The minimal nutritional requirements of collections of strains of the following seven species or varieties were examined: Bacillus cereus var. anthracis, B. cereus var. thuringiensis, B. firmus, B. lentus, B. coagulans, B. pulvifaciens and $B$. circulans. The results are summarized in Table 1 and the detailed results with each species are given below. 


\section{Bacillus cereus var. anthracis}

Six strains of Bacillus cereus var. anthracis were examined: CN $1874\left(34 \mathrm{~F}_{2}\right)$ avirulent strain from Ministry of Agriculture Laboratories, Weybridge; CN 3472 (34F $)$; CN 3482 (17 JB); CN 3518 (V8R) (these three strains from Dr M. Sterne); CN 2207 (ATCC 9660); CN 1995, a freshly isolated virulent strain from a pig. All six strains grew in the complex amino acid medium supplemented with thiamine. The requirement for thiamine was absolute in

Table 1. The minimal nutritional requirements of some species in the genus Bacillus

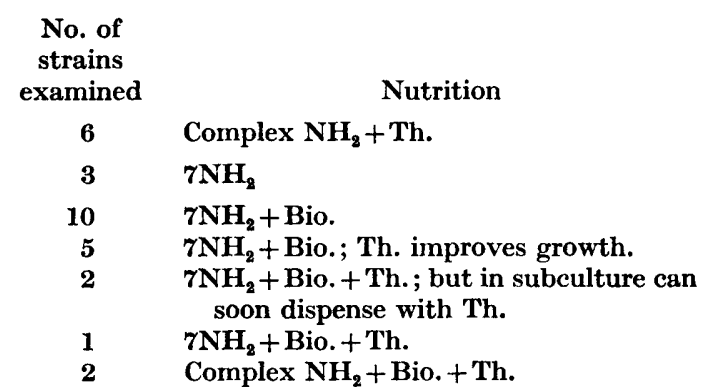

B. lentus

B. coagulans

B. pulvifaciens

B. circulans

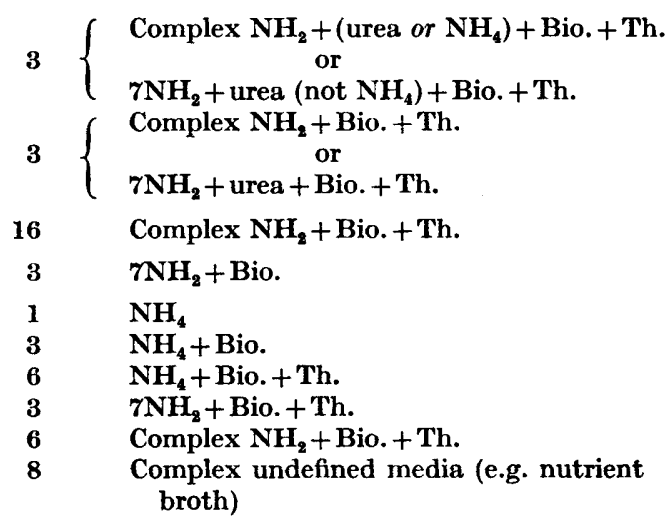

Complex $\mathrm{NH}_{2}=$ complex amino acid medium; $\mathbf{7 N H}_{2}=$ seven amino acid medium; Bio.= biotin; $\mathbf{T h} .=$ thiamine.

that no growth took place without it and no other vitamin had to be added. The efficacy of possible precursors of thiamine was not examined. Adenosine or adenylic acid sometimes improved growth, but neither was essential. Strains usually grew for one or two subcultures in the complex amino acid medium without added thiamine but they could not be maintained in serial subculture. The amino acid requirements were complex; the strains would grow on the seven amino acid medium + thiamine for a few subcultures but soon died out. They would not grow at all on the seven amino acid medium without thiamine. 


\section{Bacillus cereus var. thuringiensis}

Three strains of this insect pathogen were received from Dr W. C. Haynes: CN 3624 (NRS 996); CN 3625 (NRS 1124); CN 3626 (NRS 1328). All strains grew in the seven amino acid medium, the requirement for amino acids was absolute and no other growth factors were required.

\section{Bacillus firmus}

Twenty strains of Bacillus firmus were examined; of these strains fifteen were received from Dr Ruth Gordon, one from the American Type Culture Collection (ATCC) and one from the National Collection of Type Cultures (NCTC) and three were fresh isolates from soil. Ten of these strains-CN 2196 (ATCC 8247); CN 2544; CN 3316 (854); CN 3317 (858); CN 3318 (861); CN 3319 (855); CN 3384 (1070); CN 3386 (1122); CN 3390 (1151); CN 3389 (1149)-grew in the seven amino acid medium + biotin; the requirements for amino acids and biotin were absolute. Five other strains-CN 2936; CN 1758 (NCTC 6354); CN 3315 (783); CN 3314 (613); CN 3320 (860)-grew indifferently in the above medium, and the growth was markedly improved by the addition of thiamine. Two other strains-CN 3385 (1131); CN 3392 (1329)-required the addition of thiamine, but on serial subculture in defined medium variants were readily selected which grew in the absence of thiamine. Three other strains-CN 1654; CN 3391 (1152); CN 3387 (1147)-showed an absolute requirement for thiamine. Two of the strains $-\mathrm{CN} 3391$; $\mathrm{CN} 3387$ - had more complex amino acid requirements and would only grow with the complex amino acid medium + biotin + thiamine.

\section{Bacillus lentus}

Six strains of Bacillus lentus were examined: two strains-CN 2788; CN 2789 -were fresh isolates from soil; three strains-CN 3323 (670); CN 3321 (769); CN 3323 (749)-were received from Dr Ruth Gordon; and one strainCN 2750 (165) - from Dr T. Gibson. All strains showed an absolute requirement for amino acids + thiamine + biotin. With the complex amino acid medium three strains-CN 2750; CN 2789; CN 3323-required also the addition of urea or ammonium chloride. The growth with urea was uniformly better than with ammonium chloride. All six strains required urea in the presence of the seven amino acid medium and in this medium urea could not be replaced by ammonium chloride.

\section{Bacillus coagulans}

Sixteen strains of Bacillus coagulans were examined; one strain was from the American Type Culture Collection, ATCC 7050, and the remaining strains were fresh isolates from soil. These strains formed a nutritionally homogeneous group and all grew in the complex amino acid medium + biotin + thiamine. The amino-acid requirements were complex and the complex amino acid medium could not be replaced by the seven amino acid medium. 


\section{Bacillus pulvifaciens}

Three strains of Bacillus pulvifaciens-CN 3621 (NRS 1283); CN 3622 (NRS 1284); CN 3623 (NRS 1285)-were received from Dr W. C. Haynes. All three strains grew in the seven amino acid medium + biotin. The requirements for amino acids and biotin were absolute and no other growth factors were required.

\section{Bacillus circulans}

Twenty-seven strains of Bacillus circulans were examined; of these strains sixteen were fresh isolates from soil, nine were from Dr T. Gibson and one strain each was received from the American Type Culture Collection and from the National Collection of Type Cultures. In contrast to all the other collections of cultures representative of species within the genus Bacillus which we classified according to the criteria of Smith et al. (1952), these twenty-seven strains of $B$. circulans formed a nutritionally heterogeneous group. One strain, CN 2719, grew in the ammonia basal medium alone; added amino acids or growth factors were not required. Three strains-CN 2718; CN 2923; CN 3377 (287)--grew when biotin was added to the ammonia basal medium. Six strains-CN 2720; CN 2721; CN 2848; CN 2905; CN 2925; CN 3378 (1375)-grew when biotin and thiamine were added to the ammonia basal medium. Three strains-CN 2924; CN 2201 (ATCC 1649); CN 3375 (109)-grew in the seven amino acid medium + biotin + thiamine. Six strains-CN 2849; CN 2621; CN 2723; CN 2722; CN 2526; CN 3371 (558)--had more complex amino acid requirements and grew in the complex amino-acid medium + biotin + thiamine. The remaining eight strains-CN 2628; CN 2629; CN 1609 (NCTC 6351); CN 3370 (560); CN 3733 (261); CN 3381 (796); CN 3374 (613); CN 3379 (110)—were still more exacting in their nutrient requirements. These eight strains could be maintained in serial subculture in meat broth or in nutrient broth, they could also be maintained with some difficulty in peptone water but we were unable to grow them in defined media.

\section{DISCUSSION}

This supplementary survey of the nutrition of strains of certain species of Bacillus shows, with one exception to be discussed later, the same uniformity of nutritional patterns within species that was found in our previous work (Knight \& Proom, 1950). The six strains of $B$. anthracis, including avirulent and virulent strains, all grew on a complex mixture of amino acids + thiamine; the addition of adenosine or adenylic acid sometimes improved growth but neither was essential. These results confirm the observation of Brewer et al. (1946) with the Vollum M 36 strain. The nutritional pattern of B. anthracis was therefore different from that of the parent species $B$. cereus; the $\boldsymbol{B}$. anthracis strains showed a nutritional requirement satisfied by thiamine and they were more exacting in their amino acid requirements. This nutritional difference might be used as evidence to support the classification of $\boldsymbol{B}$. anthracis as a separate species rather than as a variety of $\boldsymbol{B}$. cereus. 
In contradistinction to the animal pathogen Bacillus anthracis, the insect pathogen $B$. thuringiensis had the same nutritional pattern as the parent species $\boldsymbol{B}$. cereus and showed a requirement for amino acids only.

The twenty strains of Bacillus firmus all showed a nutritional requirement satisfied by amino acids + biotin, but they differed amongst themselves in their requirements for thiamine. According to the strain the addition of thiamine either made little difference, improved growth or was essential. The nutritionally most exacting strain of $\boldsymbol{B}$. firmus had the nutritional pattern of the least exacting strain of $B$. lentus.

The Bacillus firmus and $\boldsymbol{B}$. lentus strains may be arranged in an order which suggests a nutritional series with the least exacting strains, requiring a simple mixture of amino acids + biotin and the most exacting a complex mixture of amino acids + urea + biotin + thiamine. In addition, the $B$. lentus strains showed a requirement satisfied by urea or by ammonia, according to the mixture of amino acids present. This gradation in nutritional pattern without the usual clear-cut differences is reflected in the difficulty in finding adequate bacteriological tests for distinguishing $\boldsymbol{B}$. firmus from $\boldsymbol{B}$. lentus. It is also in keeping with the observation made previously (Knight \& Proom, 1950) that a disproportionate number of intermediates was encountered when strains of these species were isolated from soil: of eleven isolates clearly belonging to the firmus-lentus group, only five could be allocated to one or other species and six were intermediate.

The sixteen strains of Bacillus coagulans examined formed a nutritionally homogeneous group and all grew on the complex amino acid medium + biotin + thiamine. No other growth factors were required, and without biotin and thiamine there was no growth. Some strains occasionally showed growth for a few subcultures without added biotin or thiamine but for serial subculture both substances were needed. These results differ from those reported by Cleverdon, Pelczar \& Doetsch (1949) and by Allen (1953); we have no explanation for the differences.

In previous work (Knight \& Proom, 1950) we found difficulty in determining the nutritional requirements of Bacillus firmus and B. lentus isolates, and their nutrition was described as complex. Also we were unable to replace the hydrolysed casein by a defined amino acid mixture for $\boldsymbol{B}$. coagulans isolates. These effects were not due to a missing growth factor but, it now appears, to the presence of inhibitors. Proom (1955) showed that growth inhibitory concentrations of colloidal sulphur or colloidal copper sulphide may be formed during preparation of defined media containing mixtures of amino acids. These inhibitors were responsible, for example, for the failure of cultures of Bordetella pertussis to grow in a defined amino acid medium, whereas the same strains grew in the presence of hydrolysed casein. The method of preparation of amino acid mixtures used in our previous work did not exclude the possibility of the production of these inhibitors. Some strains of Bacillus firmus, all strains of $\boldsymbol{B}$. lentus and of $\boldsymbol{B}$. coagulans were found to be inhibited by the complex amino acid medium which had been gassed with $\mathrm{H}_{2} \mathrm{~S}$ to remove traces of heavy metals and finally sterilized by autoclaving; but these organisms 
were not inhibited by the complex amino acid medium prepared in the manner described in the present paper. These species (B. firmus, B. lentus, B. coagulans) differ from others in the genus Bacillus, which are apparently not inhibited by colloidal sulphur or colloidal copper sulphide.

The nutritional requirements of the three strains of Bacillus pulvifaciens examined were satisfied by the seven amino acid medium + biotin. The nutritional requirements were therefore different from those of the other species in the morphological group 2 of the genus Bacillus.

In contrast to the strains of all other species of the genus Bacillus which we have examined, the 20 strains defined as $B$. circulans formed a nutritionally heterogeneous group. So far we have been unable to grow 8 of these strains in defined media, although they can be maintained in serial subculture on nutrient agar or in nutrient broth. The remaining strains grew when given respectively and depending on the strain, either ammonia, ammonia + biotin, ammonia + biotin + thiamine, seven amino acid medium + biotin + thiamine, or the complex amino acid medium + biotin + thiamine. The heterogeneity of the nutritional requirements of this group of isolates is in keeping with the physiological and biochemical variations observed within the $B$. circulans complex as at present defined and which has made classification and identification of members of this group the most difficult in the genus. If one is prepared to accept consistency of nutritional pattern as part of the characterization of species within the genus Bacillus, the implication is that the $B$. circulans complex is at present inadequately classified.

Our thanks are due to Miss G. M. Trim for invaluable technical assistance.

\section{REFERENCES}

Allen, M. B. (1953). The thermophilic aerobic sporeforming bacteria. Bact. Rev. $17,125$.

Brewer, C. R., McCullough, W. G., Mills, R. C., Roessler, W. C., Herbst, E. J. \& Howe, A. F. (1946). Studies on the nutritional requirements of Bacillus anthracis. Arch. Biochem. 10, 65.

Cleverdon, R. C., Pelczar, Jr., M. J. \& Doetsch, R. N. (1949). Vitamin requirements of Bacillus coagulans. J. Bact. 58, 113.

Knight, B. C. J. G. \& Proom, H. (1950). A comparative survey of the nutrition and physiology of mesophilic species in the genus Bacillus. J. gen. Microbiol. 4, 508.

Proom, H. (1955). The minimal nutritional requirements of organisms of the genus Bordetella Lopez. J. gen. Microbiol. 12, 63.

Smith, N. R., Gordon, R. E. \& Clark, F. E. (1952). Aerobic sporeforming bacteria. U.S. Dep. Agric. Agriculture Monograph no. 16. Washington, D.C. 